\section{ORIGINAL RESEARCH}

\author{
D. Ramanathan \\ B. Ghodke \\ L.J. Kim \\ D. Hallam
}

M. Herbes-Rocha

L.N. Sekhar

\title{
Endovascular Management of Cerebral Bypass Graft Problems: An Analysis of Technique and Results
}

BACKGROUND AND PURPOSE: Cerebral bypass grafts may develop generalized graft narrowing or focal stenosis during the perioperative period or later. Endovascular techniques such as PTA and stent placement of graft vessels are potential treatment options. Our objective was to review the safety, indications, technique, and results of endovascular management of graft problems.

MATERIALS AND METHODS: All patients with cerebral bypass procedures by using graft vessels from 2005 to 2009 were identified from a prospective registry and were studied retrospectively. Patient characteristics, bypass procedures, indications for endovascular interventions, graft patency, and clinical outcomes were reviewed from medical charts and imaging records.

RESULTS: A total of 79 patients underwent bypass procedures by using graft vessels. Seven patients of this group underwent endovascular interventions for the treatment of graft narrowing. Four of the 7 patients were treated for graft narrowing in the perioperative period ( $<1$ month) with PTA; and 3 of the 7 patients, for late stenosis, 2 with PTA alone and 1 with PTA followed by stent placement. All procedures were immediately successful in improving flow through the graft. In late stenosis, PTA alone provided temporary improvement followed by recurrence, whereas PTA with a stent procedure was effective in the 1 patient long term.

CoNCLUSIONS: PTA is safe and effective in the management of graft spasm in the perioperative period. For late graft stenosis, PTA alone provides only temporary respite, while PTA with stent placement may be an effective solution.

ABBREVIATIONS: ATA = anterior tibial artery; ECA = external carotid artery; $\mathrm{ECIC}=$ extracranialto intracranial; IADSA = intra-arterial digital subtraction angiography; IC = intracranial; ICA = internal carotid artery; $\mathrm{MCA}=$ middle cerebral artery; $\mathrm{mRS}=$ modified Rankin Scale; $\mathrm{PCA}=$ posterior cerebral artery; PTA = percutaneous transluminal angioplasty; RAG = radial artery graft; SVG = saphenous vein graft; VA = vertebral artery

C erebral revascularization with bypass grafts is an important tool in the treatment of complex cerebral aneurysms, cerebral ischemia, and skull base tumors. Autologous graft vessels such as RAGs or SVGs are used to perform ECIC bypasses or IC-IC bypasses. A small number of these bypass grafts may develop problems of poor flow due to vasospasm during the perioperative period ( $<30$ days) and/or focal segmental stenosis during the late follow-up period. This article studies the use of endovascular interventions in the management of bypass graft problems-both immediate and long term.

\section{Materials and Methods}

All patients who underwent cerebral bypass procedures by using graft vessels from January 2005 to December 2009 were identified for an institutional review board-approved prospective cerebrovascular registry. Medical charts were retrospectively reviewed for patient characteristics, bypass procedures, the presence of graft narrowing, indications for endovascular interventions, postprocedure patency, and clinical outcomes. Graft patency was studied from IADSA or CT angiography before and after the endovascular procedure and during

Received October 22, 2010; accepted after revision January 5, 2011.

From the Departments of Neurological Surgery (D.R., B.G., L.J.K., D.H., M.H.-R., L.N.S.) and Radiology (B.G., L.J.K., D.H., L.N.S.), University of Washington, Seattle, Washington.

Please address correspondence to Laligam N. Sekhar, MD, FACS, Department of Neurological Surgery, University of Washington, Seattle, WA 98104; e-mail: Isekhar@uw.edu http://dx.doi.org/10.3174/ajnr.A2565

follow-up care. In case of late stenosis, endovascular treatment was generally chosen when the stenosis was present in the extracranial segment of the graft. Surgical corrections were performed when the stenosis was present in the intradural portion of the graft or when the extracranial stenosis extended for a significant length of the graft. The surgical technique used in 1 patient (illustrative case 1) was previously reported. ${ }^{1}$

\section{Results}

A total of 99 patients underwent 125 cerebral revascularization procedures for the treatment of aneurysms $(n=80)$, ischemia $(n=31)$, and tumors $(n=14)$. Seventy-nine bypasses were performed by using graft vessels, which included 59 RAGs, 18 SVGs, and 2 tibial arteries. The mean follow-up period of graft bypasses was 29 months. Of this group of 79 bypasses by using graft vessels, 4 grafts developed severe vasospasm (all RAGs) during the perioperative period and 8 grafts (7 RAGs and 1 SVG) developed focal segmental stenosis ( $\geq 50 \%$ ) during the late follow-up period. All 4 grafts that developed perioperative vasospasm and 3 of the 8 grafts that developed stenosis during the late follow-up period were treated with endovascular intervention. The remaining 5 grafts with late stenosis were managed with surgical repair $(n=4$, three RAGs and 1 SVG) and periodic monitoring ( $n=1$, one RAG). Of the 4 grafts that underwent primary surgical correction, 3 had intracranial graft stenosis and 1 had nonfocal stenosis, present in a considerable portion of the extracranial segment. 
The PTA procedures performed in the perioperative period were all immediately successful in improving the flow through the graft, as evident in the completion angiograms. During the follow-up period, 2 (1 VA-to-PCA RAG for ischemia and 1 MCA-to-PCA RAG bypass for aneurysm clipping) of the 4 grafts were occluded with enlargement of collateral vessels. The other 2 bypasses (an ECA-to-MCA RAG bypass for Moyamoya disease and an ICA-to-MCA RAG bypass for aneurysm clipping) remained patent at 18 and 48 months follow-ups.

Three of the 7 patients who were treated with endovascular interventions underwent the procedure during the late follow-up period for symptomatic or severe focal stenosis. Endovascular interventions in the late stenosis group included PTA alone $(n=2)$ and PTA followed by stent placement in the stenotic graft $(n=1)$. All 3 procedures were successful immediately in improving the flow through the graft. Two of the patients who underwent only PTA developed recurrent symptoms due to restenosis, while there was no evidence of restenosis in the patient who had PTA and stent placement, as noted at 6 months' follow-up with MR angiography. The recurrent stenoses were surgically repaired by interposition graft placement after resection of the stenoses and a jump graft bypass procedure.

\section{Technique}

All PTA procedures were performed with standard transfemoral arterial access. Intravenous heparin titrated to an activated clotting time of 250-300 seconds was used for systemic anticoagulation in PTA procedures performed in the first 2 weeks postbypass surgery. In cases of late graft stenoses, patients were placed on aspirin ( $325 \mathrm{mg}$ once daily) and/or clopidogrel for at least 3 days before the procedure in addition to the heparin used during the procedure. After diagnostic angiography, parent vessel catheterization was performed with a $6 \mathrm{~F}$ guiding catheter. Following this, an Excelsior SL-10 microcatheter (Boston Scientific, Natick, Massachusetts) with a 0.014-inch Synchro microguidewire (Boston Scientific) was used to catheterize the graft vessel. The catheters were inserted beyond the site of stenosis/narrowing, and angiography was performed through the guide catheter to visualize the rest of the graft. A compliant $4 \times 10 \mathrm{~mm}$ HyperGlide balloon catheter (ev3, Irvine, California) was used for performing the dilation in most procedures. In cases of resistant stenoses, semicompliant Gateway (Boston Scientific) or Monorail Maverick (Boston Scientific) balloons were used for PTA. In perioperative patients, multiple sequential balloon dilations were performed along the entire length of the graft vessel, proceeding from distal to proximal. In focal/segmental stenosis, the PTA procedure was limited to the narrowed region of the graft. In 1 patient, a Neuroform stent (Boston Scientific) was deployed in the proximal part of the RAG bypass from the ECA to the MCA.

The balloons were gradually inflated under close visual inspection to avoid overdilation and rupture of the stenotic vessel. Special care was taken to avoid hyperinflation when using the compliant HyperGlide balloons.

There were no complications during or immediately after the endovascular interventions. There was 1 instance of an unsuccessful catheterization in a patient while attempting a repeat angioplasty procedure for a restenosis. The inability to catheterize the graft was due to a narrowing proximal to the stenosis, where a synthetic graft patch was used to repair graft injury caused by multiple arterial line insertions before harvesting the graft.

\section{Illustrative Case 1 (Patient 1)}

A 72-year-old woman presented with subarachnoid hemorrhage from a ruptured basilar tip aneurysm, which was treated by endovascular coiling. Due to coil compaction and recurrence of the aneurysm $(21 \times 19 \times 25 \mathrm{~mm})$, microsurgical clipping was planned. Intraoperatively, due to an inability to clip the aneurysm without occluding the PCA, an IC-IC bypass from the right M2 segment of the MCA to the P2 segment of the PCA was performed to enable clipping. Postoperative angiography showed excellent flow through the graft with complete occlusion of the aneurysm, and the patient recovered well from surgery (Fig 1A). However, on postoperative day 2 , the patient was noted to be worse than her preoperative condition, with somnolence and mild hemiparesis. An angiogram showed a narrowed graft with decreased flow, for which endovascular salvage was planned (Fig $1 B$ ). With the patient under general anesthesia and 0.035 -inch guidewire access, a $6 \mathrm{~F}$ Envoy guiding catheter (Cordis, Miami Lakes, Florida) was used for catheterization of the right ICA. A $4 \times 10 \mathrm{~mm} \mathrm{Hy-}$ perGlide balloon was advanced over a 0.10 Expedion guidewire (ev3) into the graft (Fig 1C). Multiple inflations of the balloon were performed to dilate the vessel at areas of narrowing. Angiograms after the procedures showed notable improvement in the flow through the graft (Fig 1D). However, there was mild narrowing in the proximal and distal portions of the graft, but further angioplasty was not attempted because of much-improved flow. At 3 months postsurgery, graft flow was sluggish. The graft was found completely occluded on IADSA obtained at 18 months, with filling of the right PCA from an enlarged right PCA (not shown). At 4-year follow-up, she is independent for activities of daily living with some gait ataxia, similar to her preoperative baseline (mRS 2).

\section{Illustrative Case 2 (Patient 6)}

A 47-year-old man presented with a subarachnoid hemorrhage from a giant left MCA aneurysm. The aneurysm was microsurgically clipped, leaving a remnant of the neck to preserve flow through distal MCA branches, which were originating from the aneurysm. The patient recovered during the following months but had significant problems with cognition, memory, and judgment. Cerebral angiography revealed a significant stenosis of the superior and major M2 branch of the MCA with significant flow compromise (Fig 2A). A singlephoton emission CT scan revealed a flow abnormality in the distribution of the superior branch of the MCA. Because no superficial temporal artery was available, a RAG bypass was performed from the ECA to the M2 branch of the MCA on the left side (Fig 2B). Eight months later, the patient developed symptoms of episodic and significant headaches along with a return of memory problems. On IADSA, the graft was homogeneously narrowed, with an irregular stenosis near the origin and proximal segment (Fig 2C). He was placed on aspirin, clopidogrel, and lovastatin and underwent endovascular intervention 3 days later. An Excelsior SL-10 preshaped $45^{\circ} \mathrm{mi}-$ crocatheter with a Synchro-14 guidewire combination was 

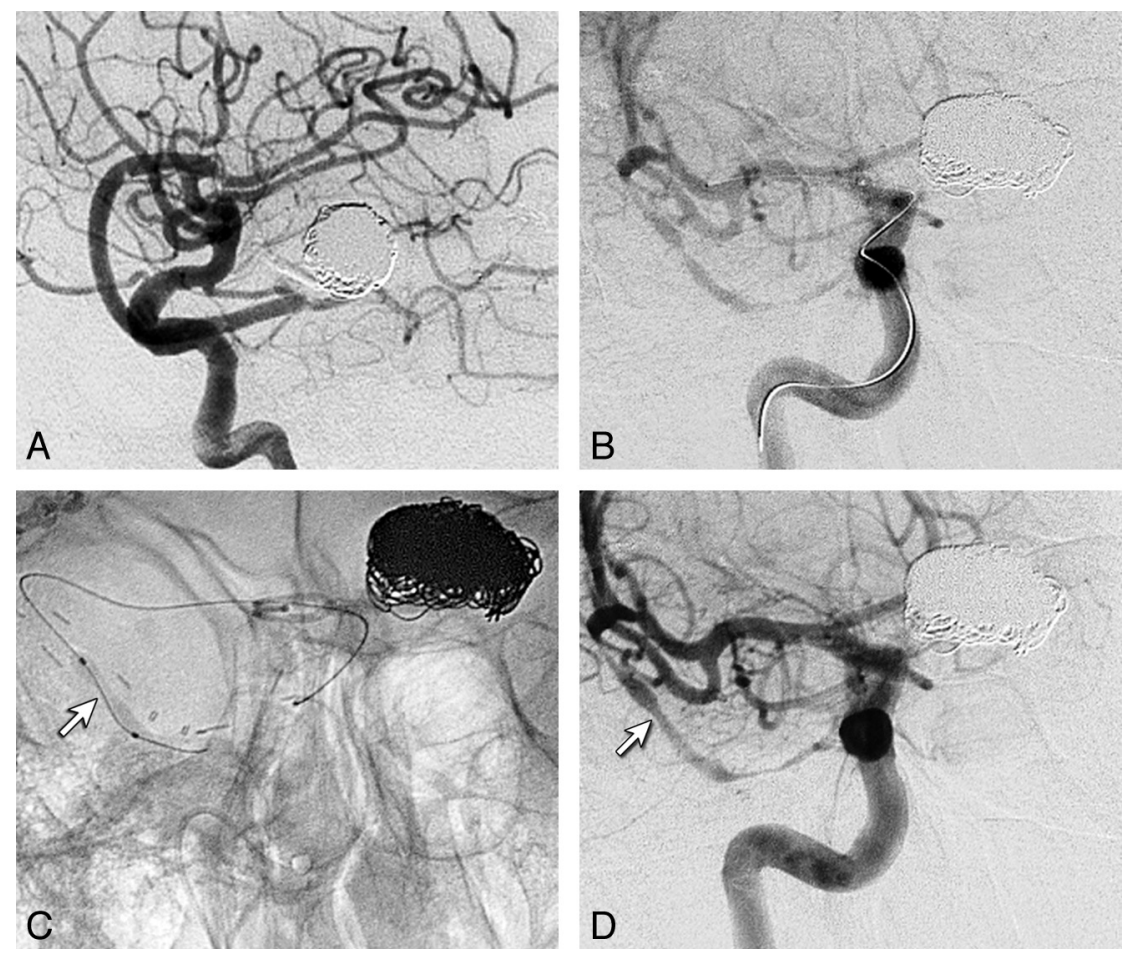

Fig 1. $A$, Postoperative angiogram (IADSA), lateral view right ICA injection, shows the IC-IC bypass from the MCA to the PCA. $B$, Angiogram (IADSA), right ICA anteroposterior view, with the catheter showing poor graft filling before the angioplasty procedure. $C$. Angiogram shows the balloon catheter (arrow) positioned in the graft across the segment of narrowing in the proximal graft. D, Post-PTA IADSA showing much better graft flow (arrow). Distal segments of the graft show narrowing.

used to access the left ECA-MCA RAG for subselective injection angiography. Then, a HyperGlide balloon catheter and an Expedion-10 guidewire combination was used to access the radial RGA, and sequential balloon dilation was performed of the proximal third to half of the RGA. Last, a Monorail Maverick $3.0 \times 20 \mathrm{~mm}$ balloon catheter was used to dilate 2 persistent stenoses in the proximal third of the RGA. Following this, a Neuroform stent $4.5 \times 30 \mathrm{~mm}$ was deployed in the proximal portion of the graft (Fig 2D). The stenosis was corrected, and there was marked improvement in the flow through the graft as noted in the completion angiograms (Fig 2E). At 3-month follow-up angiography, his graft was patent with good flow (Fig $2 F$ ), also confirmed by an MR angiography at 6 months (not shown). At 9 months' clinical follow-up after the repair procedure, the patient remains free of any symptoms (mRS 0).

\section{Discussion}

Endovascular interventions such as PTA and stent placement are commonly used in the treatment of stenoses in coronary and cerebral vasculature. PTA is also used in the treatment of cerebral vasospasm following subarachnoid hemorrhage. The use of such techniques in bypass grafts may logically be considered high-risk, given the presence of surgical anastomosis and possible graft inflammation immediately following a bypass procedure. Endovascular treatment of bypass grafts with high success rates has been described in the coronary circulation. $^{2-4}$ The incidence of vasospasm in bypass grafts has significantly decreased with the use of the pressure distention technique. ${ }^{5}$ Sekhar et $a l,{ }^{5}$ in 2001, reported 2 cases of ECIC RAG vasospasm treated with angioplasty: One was successful, and the other ruptured during the procedure. Since that initial report, the technology of endovascular devices and catheters has advanced significantly and 1 other successful salvage of a bypass graft with PTA has been reported. ${ }^{6}$ In this series of patients, there were no complications of endovascular interventions.

In the immediate postprocedural period, all PTA procedures were successful in relieving vasospasm and improving graft flow. During the medium-to-late follow-up period, focal or segmental stenosis of high-flow grafts is generally due to intimal hyperplasia. By extrapolating the evidence from the management of similar pathology in the coronary vasculature, it is logical to expect stent procedures to be more effective than PTA alone in treating the late ECIC graft stenoses. ${ }^{4,7-11}$ Supporting this hypothesis, both patients who were treated with PTA alone for late RAG stenosis had recurrences after 3 and 5 months (patients 5 and 7). On the other hand, PTA with deployment of a stent in a stenotic RAG (in 1 patient) was effective, as noted with a 6-month follow-up (illustrative case 1). Long-term follow-up after stent placement, however, is needed because the occurrence of in-stent restenosis is not infrequent with the use of bare metal stents.

Any attempt to repair the graft either surgically or endovascularly should be reserved for progressive stenosis, highgrade stenosis $(>50 \%)$, or symptomatic stenosis. The possibility of spontaneous resolution of minimal graft stenosis, especially with good control of risk factors such as hypertension and hyperlipidemia, should be considered. Endovascular interventions in this group were limited to late stenoses occurring in the extracranial segments of the graft, whereas surgical corrections were performed for intracranial graft stenoses. In severely stenotic vessels, when there is genuine difficulty in accessing the graft vessel lumen, it may be prudent to abort the 

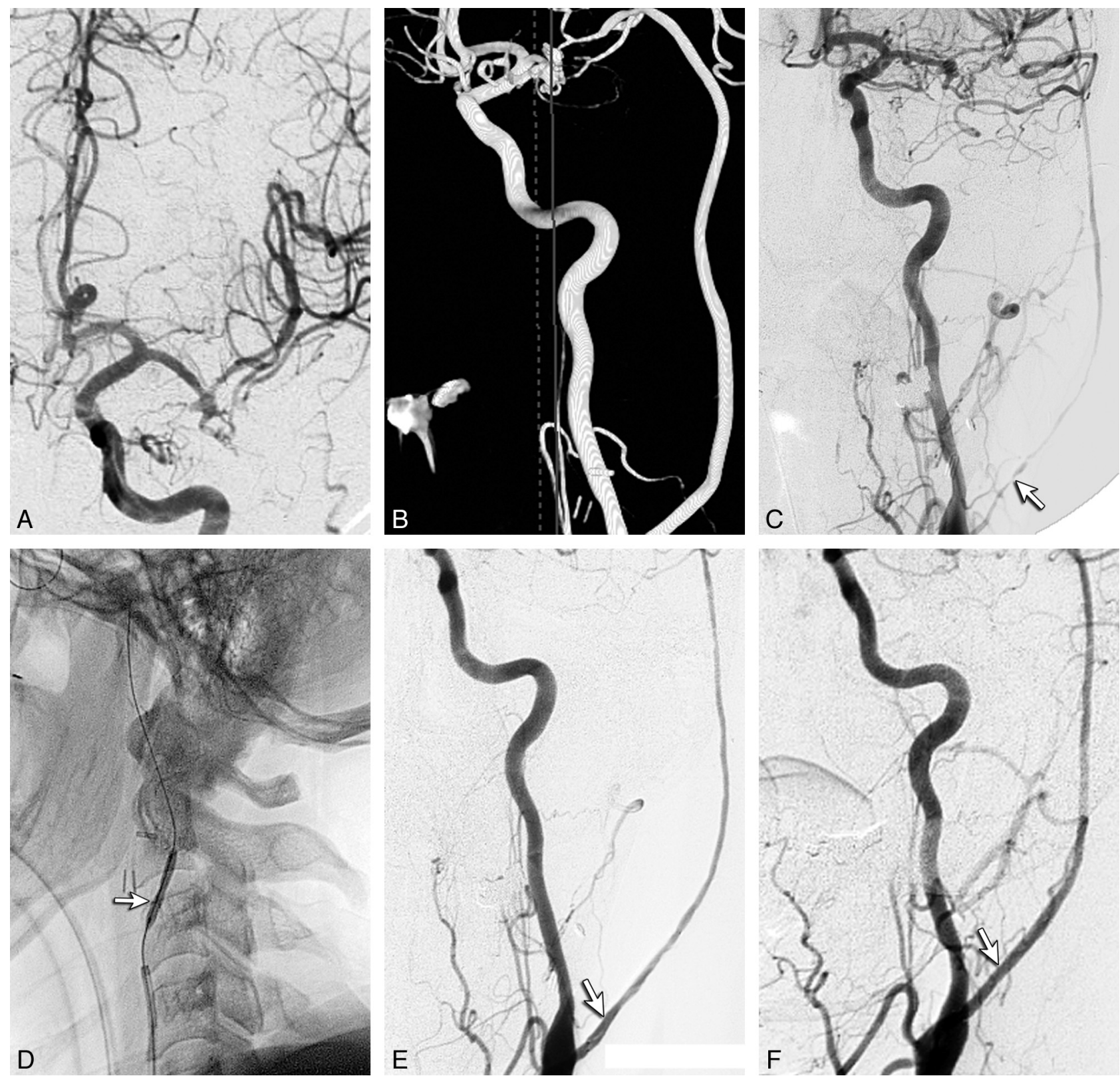

Fig 2. A, Angiogram (IADSA), ICA anteroposterior view, status postclipping of a giant MCA aneurysm shows the stenotic MCA branches-superior and inferior. $B$, Angiographic $3 D$ reconstruction shows the RAG bypass on the left side from the ECA to the MCA M2, immediately postsurgery. $C$, Angiogram (IADSA), ICA injection, anteroposterior view, 8 months postoperatively, shows the poor flow in the graft with a stenosis in the proximal fourth of the graft (arrow). $D$, Angiogram, lateral view left common carotid injection, shows the HyperGlide balloon catheter (arrow) with the Envoy catheter in the graft vessel. E, Angiogram (IADSA), ICA injection, anteroposterior oblique view, immediately after angioplasty and stent placement (arrow), shows good filling of the bypass graft. F, Angiogram (IADSA), common carotid anteroposterior oblique view 3 months after angioplasty and stent placement, with the arrow showing the stent in place and a much-improved flow.

procedure and defer to surgical treatment options. Surgical repair is also advised for any stenosis refractory to endovascular intervention or when the stenosis is nonfocal and present in a considerable portion of the graft. Review of this series of patients adds evidence to the safety and role of endovascular interventions both in alleviation of graft spasm in the perioperative period and for the management of stenosis during late follow-up.

\section{Conclusions}

Endovascular techniques such as PTA and stent placement are safe to perform in ECIC bypass graft vessels in the extracranial segment of the graft. In the perioperative period, PTA of ex- tracranial segments of the graft is safe and effective in improving poor graft flow. For focal stenoses of the grafts occurring during the follow-up period, PTA alone may only provide a temporary respite, whereas PTA along with stent placement seems to be an effective option.

Disclosures: Daniel K. Hallam, Consultant. Penumbra; Concentric Medical, Details: Honoraria for users' meetings. Louis Kim, Ownership Interest: SPI Surgical, Inc, Details: Medical device start-up company involved with surgical robotics.

\section{References}

1. Ramanathan D, Ciporen J, Ghodke B, et al. Treatment of coil embolization failed recurrent giant basilar tip aneurysms with bypass and surgical occlusion. J Neurointerv Surg 2010;2:237 
2. Hung WC, Wu CJ, Yip HK, et al. Percutaneous transluminal angioplasty to left internal mammary artery grafts: immediate and long-term clinical outcomes. Chang Gung Med J 2007;30:235-41

3. Sharma $\mathrm{AK}$, Ajani $\mathrm{AE}$, Garg $\mathrm{N}$, et al. Percutaneous interventions in radial artery grafts: clinical and angiographic outcomes. Catheter Cardiovasc Interv 2003;59:172-75

4. Sirnes PA, Golf S, Myreng Y, et al. Stenting in Chronic Coronary Occlusion (SICCO): a randomized, controlled trial of adding stent implantation after successful angioplasty. J Am Coll Cardiol 1996;28:1444-51

5. Sekhar LN, Duff JM, Kalavakonda C, et al. Cerebral revascularization using radial artery grafts for the treatment of complex intracranial aneurysms: techniques and outcomes for 17 patients. Neurosurgery 2001;49:646-58, discussion $658-59$

6. Natarajan SK, Hauck EF, Hopkins LN, et al. Endovascular management of symptomatic spasm of radial artery bypass graft: technical case report. $\mathrm{Neu}$ rosurgery, 2010;67:794-98, discussion 798
7. Hoher M, Wohrle J, Grebe OC, et al. A randomized trial of elective stenting after balloon recanalization of chronic total occlusions. J Am Coll Cardio 1999;34:722-29

8. Kiemeneij F, Serruys PW, Macaya C, et al. Continued benefit of coronary stenting versus balloon angioplasty: five-year clinical follow-up of Benestent-I trial. J Am Coll Cardiol 2001;37:1598-603

9. Prasad A, Rihal CS, Lennon RJ, et al. Trends in outcomes after percutaneous coronary intervention for chronic total occlusions: a 25-year experience from the Mayo Clinic. J Am Coll Cardiol 2007;49:1611-18

10. Rodriguez A, Rodriguez Alemparte M, Fernandez Pereira C, et al. Latin American randomized trial of balloon angioplasty vs coronary stenting for small vessels (LASMAL): immediate and long-term results. Am J Med 2005;118:743-51

11. Rubartelli P, Verna E, Niccoli L, et al. Coronary stent implantation is superior to balloon angioplasty for chronic coronary occlusions: six-year clinical follow-up of the GISSOC trial. J Am Coll Cardiol 2003;41:1488-92 